\title{
AN EXTENDED FINITE ELEMENT METHOD APPLIED TO LEVITATED DROPLET PROBLEMS
}

\author{
PATRICK ESSER, JÖRG GRANDE, ARNOLD REUSKEN*
}

\begin{abstract}
We consider a standard model for incompressible two-phase flows in which a localized force at the interface describes the effect of surface tension. If a level set method is applied then the approximation of the interface is in general not aligned with the triangulation. This causes severe difficulties w.r.t. the discretization and often results in large spurious velocities. In this paper we reconsider a (modified) extended finite element method (XFEM), which in previous papers has been investigated for relatively simple two-phase flow model problems, and apply it to a physically realistic levitated droplet problem. The results show that due to the extension of the standard FE space one obtains much better results in particular for large interface tension coefficients. Furthermore, a certain cut-off technique results in better efficiency without sacrificing accuracy.
\end{abstract}

Key words. finite element, surface tension, spurious velocities, level set method, two-phase flow

AMS subject classifications. 65M60, 65N15, 65N30, 76D45, 76T99

1. Introduction. We consider a standard model for incompressible two-phase flows in which a localized force at the interface describes the effect of surface tension. If a level set method is applied then the interface, which is implicitly given by the zero level of the level set function, is in general not aligned with the triangulation that is used in the discretization of the flow problem. This non-alignment causes severe difficulties w.r.t. the discretization of the localized surface tension force and the discretization of the flow variables. In cases with large surface tension forces the pressure has a large jump across the interface. In standard finite element spaces, due to the non-alignment, the functions are continuous across the interface and thus not appropriate for the approximation of the discontinuous pressure. In many simulations these effects cause large oscillations of the velocity close to the interface, so-called spurious velocities.

Extended finite element spaces (XFEM) as presented in $[12,2]$ allow a much better (even optimal) approximation of the discontinuous pressure. In the previous paper [8] we analyzed this XFEM approach for model problems, like for example a quiescent droplet with surface tension ( $\mathbf{u}=0$ and a piecewise constant pressure). It was shown both by theoretical analysis and numerical experiments that with an XFEM discretization the size of spurious velocities can be reduced substantially. In another paper [16] we derived approximation error bounds for this method and introduced a variant in which discontinuous basis functions that were originally added in the extended finite element space are left out if they have a "very small" support. This cutoff technique leads to a modified XFE space with the same (optimal) approximation quality as the original XFE space but results in linear systems that are easier to solve.

In the present paper we reconsider this (modified) XFEM approach and apply it to a physically realistic levitated droplet problem. We do not know of any other literature in which this type of finite element discretization (with optimal approximation error properties) is combined with a level set interface capturing technique and applied in a three-dimensional simulation of a physically realistic two-phase flow problem. The results presented in the paper lead to the following two main conclusions. Firstly, the

*Institut für Geometrie und Praktische Mathematik, RWTH-Aachen University, D-52056 Aachen, Germany; email: reusken@igpm.rwth-aachen.de This work was supported by the German Research Foundation through SFB 540. 
extension of the standard linear finite element space for the pressure discretization leads to a significant improvement. This improvement increases for larger interface tension coefficients. For example, for a toluol-water system we obtain satisfactory results using the XFE pressure discretization, whereas for the standard linear finite element pressure space on the same triangulation the simulation does not yield physically realistic results. Secondly, the use of a cut-off technique in the XFE space, i.e., neglecting additional basis functions with "very small" support (as explained below), significantly improves the converge rates of the iterative solvers used. This cut-off technique is such that the good (even optimal) discretization quality is maintained. Thus, we can compute accurate discrete solutions with (much) less computational costs.

2. Problem formulation. Let $\Omega \subset \mathbb{R}^{3}$ be a domain containing two different immiscible incompressible phases. The time dependent subdomains containing the two phases are denoted by $\Omega_{1}(t)$ and $\Omega_{2}(t)$ with $\bar{\Omega}=\bar{\Omega}_{1} \cup \bar{\Omega}_{2}$ and $\Omega_{1} \cap \Omega_{2}=\emptyset$. We assume that $\Omega_{1}$ and $\Omega_{2}$ are connected and $\partial \Omega_{1} \cap \partial \Omega=\emptyset$ (i. e., $\Omega_{1}$ is completely contained in $\Omega)$. The interface is denoted by $\Gamma(t)=\bar{\Omega}_{1}(t) \cap \bar{\Omega}_{2}(t)$. The standard model for describing incompressible two-phase flows consists of the Navier-Stokes equations in the subdomains with the coupling condition

$$
[\boldsymbol{\sigma} \mathbf{n}]_{\Gamma}=\tau \mathcal{K} \mathbf{n}
$$

at the interface, i.e., the surface tension balances the jump of the normal stress on the interface. We use the notation $[v]_{\Gamma}$ for the jump of $v$ across $\Gamma, \mathbf{n}=\mathbf{n}_{\Gamma}$ is the unit normal at the interface $\Gamma$ (pointing from $\Omega_{1}$ into $\Omega_{2}$ ), $\mathcal{K}$ the curvature of $\Gamma$ and $\sigma$ the stress tensor defined by

$$
\boldsymbol{\sigma}=-p \mathbf{I}+\mu \mathbf{D}(\mathbf{u}), \quad \mathbf{D}(\mathbf{u})=\nabla \mathbf{u}+(\nabla \mathbf{u})^{T},
$$

with $p=p(\mathbf{x}, t)$ the pressure, $\mathbf{u}=\mathbf{u}(\mathbf{x}, t)$ the velocity and $\mu$ the viscosity. We assume continuity of $\mathbf{u}$ across the interface. Combined with the conservation laws for mass and momentum we obtain the following standard model, cf. for example [14, 15, 20, 19],

$$
\begin{aligned}
& \left\{\begin{array}{ll}
\rho_{i} \mathbf{u}_{t}-\operatorname{div}\left(\mu_{i} \mathbf{D}(\mathbf{u})\right)+\rho_{i}(\mathbf{u} \cdot \nabla) \mathbf{u}+\nabla p=\rho_{i} \mathbf{g} & \text { in } \Omega_{i} \times[0, T] \\
\operatorname{div} \mathbf{u}=0 & \text { in } \Omega_{i} \times[0, T]
\end{array} \text { for } i=1,2\right.
\end{aligned}
$$

The constants $\mu_{i}, \rho_{i}$ denote viscosity and density in the subdomain $\Omega_{i}, i=1,2$, and $\mathrm{g}$ is an external volume force (gravity). To make this problem well-posed we need suitable boundary conditions for $\mathbf{u}$ and an initial condition $\mathbf{u}(\mathbf{x}, 0)$.

The location of the interface $\Gamma(t)$ is in general unknown and is coupled to the local flow field which transports the interface. Various approaches are used for approximating the interface. Most of these can be classified as either front-tracking or front-capturing techniques. In this paper we use a level set method $[4,13,18]$ for capturing the interface.

The two Navier-Stokes equations in $\Omega_{i}, i=1,2$, in (2.1) together with the interfacial condition (2.2) can be reformulated in one Navier-Stokes equation on the whole domain $\Omega$ with an additional force term localized at the interface, the so called continuum surface force (CSF) model $[3,4]$. Combination of the CSF approach with the 
level set method leads to the following model for the two-phase problem in $\Omega \times[0, T]$ :

$$
\begin{aligned}
\rho(\phi)\left(\frac{\partial \mathbf{u}}{\partial t}+(\mathbf{u} \cdot \nabla) \mathbf{u}\right) & =-\nabla p+\rho(\phi) \mathbf{g}+\operatorname{div}(\mu(\phi) \mathbf{D}(\mathbf{u}))+\tau \mathcal{K} \delta_{\Gamma} \mathbf{n}_{\Gamma} \\
\operatorname{div} \mathbf{u} & =0 \\
\phi_{t}+\mathbf{u} \cdot \nabla \phi & =0 .
\end{aligned}
$$

This model has to be interpreted in a suitable weak sense. Appropriate initial and boundary conditions have to be added to make it well-posed. This model is used for the levitated droplet problem in section 5 .

3. An extended FE method for pressure discretization. In this section we briefly recall the extended FE (XFE) space discussed in [8] and a modified space presented in [16]. The latter space is used for the discretization of the pressure variable in our two-phase levitated droplet problem in section 5 .

Let $\mathcal{T}_{h}$ be a triangulation of the domain $\Omega$ consisting of tetrahedra and let

$$
Q_{h}=\left\{q \in C(\Omega)|q|_{T} \in \mathcal{P}_{1} \text { for all } T \in \mathcal{T}_{h}\right\}
$$

be the standard finite element space of continuous piecewise linear functions. We define the index set $\mathcal{J}=\{1, \ldots, n\}$, where $n=\operatorname{dim} Q_{h}$ is the number of degrees of freedom. Let $\mathcal{B}:=\left\{q_{j}\right\}_{j=1}^{n}$ be the nodal basis of $Q_{h}$, i. e. $q_{j}\left(\mathbf{x}_{i}\right)=\delta_{i, j}$ for $i, j \in \mathcal{J}$ where $\mathbf{x}_{i} \in \mathbb{R}^{3}$ denotes the spatial coordinate of the $i$-th degree of freedom.

The idea of the XFEM method is to enrich the original finite element space $Q_{h}$ by additional basis functions $q_{j}^{X}$ for $j \in \mathcal{J}^{\prime}$ where $\mathcal{J}^{\prime} \subset \mathcal{J}$ is a given index set. An additional basis function $q_{j}^{X}$ is constructed by multiplying the original nodal basis function $q_{j}$ by a so called enrichment function $\Phi_{j}$ :

$$
q_{j}^{X}(\mathbf{x}):=q_{j}(\mathbf{x}) \Phi_{j}(\mathbf{x}) .
$$

This enrichment yields the extended finite element space

$$
Q_{h}^{X}:=\operatorname{span}\left(\left\{q_{j}\right\}_{j \in \mathcal{J}} \cup\left\{q_{j}^{X}\right\}_{j \in \mathcal{J}^{\prime}}\right) .
$$

This idea was introduced in [12] and further developed in [2] for different kinds of discontinuities (kinks, jumps), which may also intersect or branch. The choice of the enrichment function depends on the type of discontinuity. For representing jumps the Heaviside function is proposed to construct appropriate enrichment functions. Basis functions with kinks can be obtained by using the distance function as enrichment function.

In our case the finite element space $Q_{h}$ is enriched by discontinuous basis functions $q_{j}^{X}$ for $j \in \mathcal{J}^{\prime}=\mathcal{J}_{\Gamma}:=\left\{j \in \mathcal{J} \mid\right.$ meas $\left._{2}\left(\Gamma \cap \operatorname{supp} q_{j}\right)>0\right\}$, as discontinuities in the pressure only occur at the interface. Let $d: \Omega \rightarrow \mathbb{R}$ be the signed distance function (or an approximation to it) with $d$ negative in $\Omega_{1}$ and positive in $\Omega_{2}$. In our applications the discretization of the level set function $\varphi$ is used for $d$. Then by means of the Heaviside function $H$ we define

$$
H_{\Gamma}(\mathbf{x}):=H(d(\mathbf{x}))= \begin{cases}0 & \mathbf{x} \in \Omega_{1} \cup \Gamma, \\ 1 & \mathbf{x} \in \Omega_{2} .\end{cases}
$$

As we are interested in functions with a jump across the interface we define the enrichment function

$$
\Phi_{j}^{H}(\mathbf{x}):=H_{\Gamma}(\mathbf{x})-H_{\Gamma}\left(\mathbf{x}_{j}\right)
$$




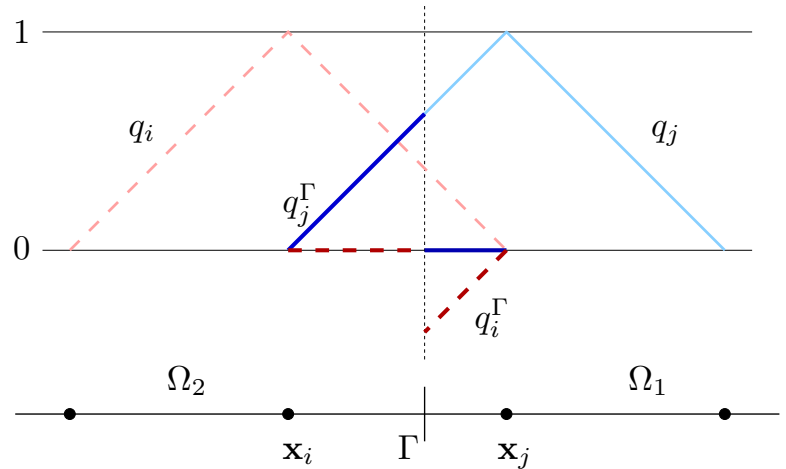

FIG. 3.1. Extended finite element basis functions $q_{i}, q_{i}^{\Gamma}$ (dashed) and $q_{j}, q_{j}^{\Gamma}$ (solid) for $1 D$ case.

and a corresponding function $q_{j}^{X}:=q_{j} \cdot \Phi_{j}^{H}, j \in \mathcal{J}^{\prime}$. The second term in the definition of $\Phi_{j}^{H}$ is constant and may be omitted (as it does not introduce new functions in the function space), but ensures the nice property $q_{j}^{X}\left(\mathbf{x}_{i}\right)=0$, i.e. $q_{j}^{X}$ vanishes in all degrees of freedom. As a consequence, we have

$$
\operatorname{supp} q_{j}^{X} \subset\left(\operatorname{supp} q_{j} \cap \bigcup_{T \in \mathcal{T}_{h}^{\Gamma}} T\right),
$$

where $\mathcal{T}_{h}^{\Gamma}=\left\{T \in \mathcal{T}_{h} \mid \operatorname{meas}_{2}(T \cap \Gamma)>0\right\}$. Thus $q_{j}^{X} \equiv 0$ in all $T$ with $T \notin \mathcal{T}_{h}^{\Gamma}$.

In the following we will use the notation $q_{j}^{\Gamma}:=q_{j} \Phi_{j}^{H}$ and

$$
Q_{h}^{\Gamma}:=\operatorname{span}\left(\left\{q_{j} \mid j \in \mathcal{J}\right\} \cup\left\{q_{j}^{\Gamma} \mid j \in \mathcal{J}_{\Gamma}\right\}\right)
$$

to emphasize that the extended finite element space $Q_{h}^{\Gamma}$ depends on the location of the interface $\Gamma$. In particular the dimension of $Q_{h}^{\Gamma}$ may change if the interface is moved. The shape of the extended basis functions for the 1D case is sketched in figure 3.1.

Note that $Q_{h}^{\Gamma}$ can also be characterized by the following property: $q \in Q_{h}^{\Gamma}$ if and only if there exist functions $q_{1}, q_{2} \in Q_{h}$ such that $\left.q\right|_{\Omega_{i}}=\left.q_{i}\right|_{\Omega_{i}}, i=1,2$.

In [16] we derived optimal approximation error bounds, both in the $L^{2}$ - and $H^{1}$ norm, for this XFE space. For example, for $p \in L^{2}(\Omega)$ with $p_{\mid \Omega_{i}} \in H^{1}\left(\Omega_{i}\right), i=1,2$, we have

$$
\inf _{q_{h} \in Q_{h}^{\Gamma}}\left\|q_{h}-p\right\|_{L^{2}} \leq \operatorname{ch}\|p\|_{1, \Omega_{1} \cup \Omega_{2}} .
$$

In [16] we introduced a modified XFE space as follows. We fix a positive constant $\hat{c}$ and define $\mathcal{J}_{\gamma} \subset \mathcal{J}_{\Gamma}$ to be the index set such that $j \in \mathcal{J}_{\gamma}$ iff

$$
\left\|q_{j}^{\Gamma}\right\|_{L^{2}(T)} \geq \hat{c} h_{T}^{2 \frac{1}{2}} \quad \text { for a } T \subset \operatorname{supp}\left(q_{j}\right) .
$$

We define the modified XFE space $Q_{h}^{\gamma} \subset Q_{h}^{\Gamma}$ by

$$
Q_{h}^{\gamma}:=\operatorname{span}\left(\left\{q_{j} \mid j \in \mathcal{J}\right\} \cup\left\{q_{j}^{\Gamma} \mid j \in \mathcal{J}_{\gamma}\right\}\right) .
$$

Note that in this space discontinuous basis functions $q_{j}^{\Gamma} \in Q_{h}^{\Gamma}$ with "very small support" (as quantified in (3.5)) are deleted. The criterion (3.5) is such that for a fixed $\hat{c}>0$ the optimal approximation result in (3.4) still holds with the space $Q_{h}^{\Gamma}$ replaced 
by the smaller space $Q_{h}^{\gamma}$, cf. [16]. Furthermore, note that for $\hat{c}=0$ we have $Q_{h}^{\gamma}=Q_{h}^{\Gamma}$ and for $\hat{c} \rightarrow \infty$ the space $Q_{h}^{\gamma}$ equals the standard space of continuous piecewise linears, i.e., $Q_{h}^{\gamma}=Q_{h}$. In our experiments we use $\hat{c}=\mathcal{O}(1)$. An important advantage of the smaller space $Q_{h}^{\gamma}$ compared to $Q_{h}^{\Gamma}$ is that the linear systems induced by $Q_{h}^{\gamma}$ turn out to be easier to solve, cf. section 5 .

REMARK 1. An interesting (open) problem is the LBB stability of the $\mathbf{V}_{h}-Q_{h}^{\Gamma}$ finite element pair ( $\mathbf{V}_{h}$ : continuous piecewise quadratics). Numerical experiments given in [16] indicate that the LBB constant

$$
C_{h, L B B}=\inf _{p_{h} \in Q_{h}^{\Gamma}} \sup _{\mathbf{v} \in \mathbf{V}_{h}} \frac{\left(\operatorname{div} \mathbf{v}_{h}, p_{h}\right)_{L^{2}}}{\left\|\nabla \mathbf{v}_{h}\right\|_{L^{2}}\left\|p_{h}\right\|_{L^{2}}}
$$

is (much) smaller for the standard XFE space $Q_{h}^{\Gamma}$ as for the modified (smaller) space $Q_{h}^{\gamma}$ with $\hat{c}=\mathcal{O}(1)$. This implies better stability properties for the space $Q_{h}^{\gamma}$ than for the original XFE space $Q_{h}^{\Gamma}$. A theoretical analysis of this stability issue is still lacking.

Let $M$ be the mass matrix in the space $Q_{h}^{\Gamma}$ w.r.t. $\left\{q_{j} \mid j \in \mathcal{J}\right\} \cup\left\{q_{j}^{\Gamma} \mid j \in \mathcal{J}_{\Gamma}\right\}$ and $D=\operatorname{diag}(M)$. In [16] it is proved that the spectral condition number of $D^{-1} M$ is uniformly bounded with respect to both $h$ and the supports of the basis functions $q_{j}^{\Gamma}$. This immediately implies a similar result for $Q_{h}^{\gamma}$.

4. Computational scheme. The main topic of this paper is the use of the modified XFE space $Q_{h}^{\gamma}$ for the discretization of the discontinuous pressure variable in two-phase incompressible flow problems. In this section we outline other components of our solver.

Spatial discretization. The spatial discretization is based on a hierarchy of tetrahedral grids. These grids are constructed in such a way that they are consistent (no hanging nodes) and that the hierarchy of triangulations is stable, [7]. An important property is that local refinement and coarsening are easy to realize. The finite element pair $\mathbf{V}_{h}-Q_{h}^{\gamma}$, with $\mathbf{V}_{h}$ the space of continuous piecewise quadratics, is used for velocity and pressure discretization. For discretization of the level set equation we use piecewise quadratic finite elements combined with streamline-diffusion stabilization.

Laplace-Beltrami discretization of $f_{\Gamma}$. In the weak form the localized surface tension force in (2.3) takes the form

$$
f_{\Gamma}(\mathbf{v})=\tau \int_{\Gamma} \mathcal{K} \mathbf{n}_{\Gamma} \cdot \mathbf{v} d s, \quad \mathbf{v} \in\left(H^{1}(\Omega)\right)^{3} .
$$

An accurate discretization of this force functional is crucial for the quality of the solver. We use a technique based on a Laplace-Beltrami representation of the curvature $\mathcal{K}$. Here we outline the main idea, further details can be found in [9]. For the discretization of the functional in (4.1) we make an approximate reconstruction of the implicitly given zero level of the discrete level set function $\phi_{h}$. The latter is a piecewise quadratic function on $\mathcal{T}_{h}$. We introduce one further regular refinement of $\mathcal{T}_{h}$, resulting in $\mathcal{T}_{h}^{\prime}$. Let $I\left(\phi_{h}\right)$ be the continuous piecewise linear function on $\mathcal{T}_{h}^{\prime}$ which interpolates $\phi_{h}$ at all vertices of all tetrahedra in $\mathcal{T}_{h}^{\prime}$. The approximation of the interface $\Gamma$ is defined by

$$
\Gamma_{h}:=\left\{\mathbf{x} \in \Omega \mid I\left(\phi_{h}\right)(\mathbf{x})=0\right\}
$$

and consists of piecewise planar segments, which are either triangles or quadrilaterals. 
Let $\nabla_{\Gamma}\left(\nabla_{\Gamma_{h}}\right)$ be the tangential gradient along $\Gamma\left(\Gamma_{h}\right)$ and $\Delta_{\Gamma}:=\nabla_{\Gamma} \cdot \nabla_{\Gamma}$ the LaplaceBeltrami operator. From differential geometry we have

$$
\int_{\Gamma} \mathcal{K} \mathbf{n}_{\Gamma} \cdot \mathbf{v} d s=-\int_{\Gamma}\left(\Delta_{\Gamma} \operatorname{id}_{\Gamma}\right) \cdot \mathbf{v} d s=\int_{\Gamma} \nabla_{\Gamma} \operatorname{id}_{\Gamma} \cdot \nabla_{\Gamma} \mathbf{v} d s
$$

with $\operatorname{id}_{\Gamma}$ the identity $\Gamma \rightarrow \mathbb{R}^{3}$, i.e., the coordinate vector on $\Gamma$. The scalar product on the right-hand side denotes the row-wise scalar product followed by summation as made precise in (4.4) below. Define

$$
\tilde{\mathbf{n}}_{h}(\mathbf{x}):=\frac{\nabla \phi_{h}(\mathbf{x})}{\left\|\nabla \phi_{h}(\mathbf{x})\right\|}, \quad \tilde{\mathbf{P}}_{h}(\mathbf{x}):=\mathbf{I}-\tilde{\mathbf{n}}_{h}(\mathbf{x}) \tilde{\mathbf{n}}_{h}(\mathbf{x})^{T}, \quad \mathbf{x} \in \Gamma_{h}, \mathbf{x} \text { not on an edge. }
$$

Based on the result in (4.3) the discrete surface tension functional is given by

$$
\tilde{f}_{\Gamma_{h}}\left(\mathbf{v}_{h}\right)=\tau \sum_{i=1}^{3} \int_{\Gamma_{h}} \tilde{\mathbf{P}}_{h}(\mathbf{x}) \mathbf{e}_{i} \cdot \nabla_{\Gamma_{h}}\left(\mathbf{v}_{h}\right)_{i} d s, \quad \mathbf{v}_{h} \in \mathbf{V}_{h},
$$

with $\mathbf{e}_{i}$ the $i$-th basis vector in $\mathbb{R}^{3}$ and $\left(\mathbf{v}_{h}\right)_{i}$ the $i$-th component of $\mathbf{v}_{h}$. The implementation of this functional requires (numerical) integration of smooth functions over the planar segments (triangles or quadrilaterals) of $\Gamma_{h}$. Note that in this approach there is no numerical regularization (or smoothing) parameter and thus it can be classified as a "sharp interface" technique. An error analysis for this surface tension discretization method is given in [9].

Time discretization. For the time discretization we apply an implicit one-step scheme to the coupled system (2.3). We use a simple $\theta$-scheme $(\theta=1$ : implicit Euler; $\theta=\frac{1}{2}$ : Crank-Nicolson). Per time step an iterative fixed point strategy decouples the discrete system for the level set unknowns from the discrete Navier-Stokes flow problem.

Reparametrization. For numerical and algorithmic purposes it is advantageous to keep the level set function close to a signed distance function during the time evolution. To realize this a reparametrization technique is needed. We apply a variant of the fast marching method $[11,18]$.

Iterative solvers. In each time step a discrete Navier-Stokes problem and a discrete level set equation must be solved. For the latter we use the GMRES method with a Gauss-Seidel preconditioner. The discrete Navier-Stokes equation are linearized using a relaxed defect correction algorithm given in [21]. This linearization results in Oseen problems of the form

$$
\left(\begin{array}{cc}
\mathbf{A} & \mathbf{B}^{T} \\
\mathbf{B} & 0
\end{array}\right)\left(\begin{array}{l}
\mathbf{v} \\
\mathbf{q}
\end{array}\right)=\left(\begin{array}{l}
\mathbf{r}_{1} \\
\mathbf{r}_{2}
\end{array}\right)
$$

For the iterative solution of these Oseen equations we apply the preconditioned generalized conjugate residual method (GCR), cf. [17]. This Krylov subspace method allows the use of a variable preconditioner. We use a block-preconditioner of the form

$$
\mathbf{P}=\left(\begin{array}{cc}
\mathbf{Q}_{A} & 0 \\
\mathbf{B} & \mathbf{Q}_{S}
\end{array}\right)
$$

where $\mathbf{Q}_{A}$ is a preconditioner of the $\mathbf{A}$-block and $\mathbf{Q}_{S}$ a preconditioner for the Schur complement $\mathbf{S}:=\mathbf{B} \mathbf{A}^{-1} \mathbf{B}^{T}$. For $\mathbf{Q}_{A}$ we use one standard multigrid $V$-cycle iteration 


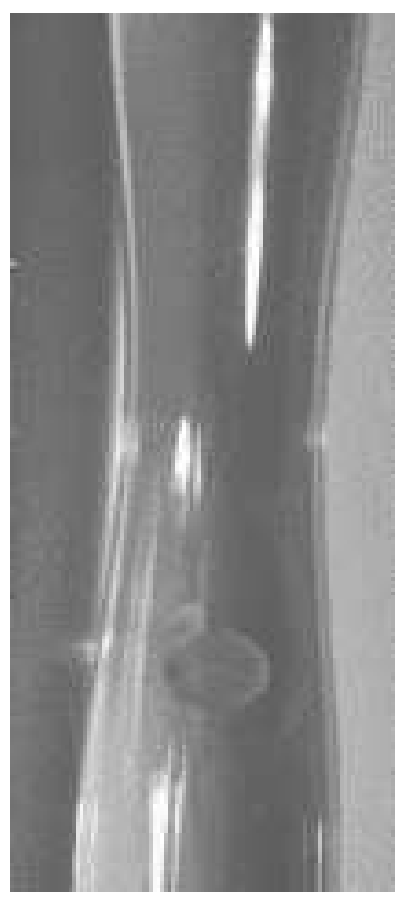

FIG. 5.1. Measuring cell with levitated toluol droplet in downward flowing water $50 \mathrm{~mm}$

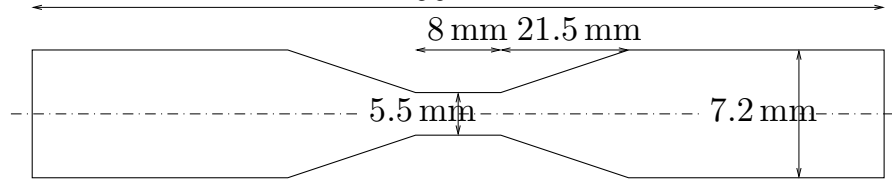

FIG. 5.2. Dimensions of the measuring cell

for the discrete diffusion-convection-reaction equations in the $\mathbf{A}$-block. For $\mathbf{Q}_{S}$ we use the scaled $B F B^{T}$-preconditioner

$$
\mathbf{Q}_{S}^{-1}=\mathbf{M}_{Q}^{-1 / 2}\left(\tilde{\mathbf{B}} \tilde{\mathbf{B}}^{T}\right)^{-1} \tilde{\mathbf{B}} \tilde{\mathbf{A}} \tilde{\mathbf{B}}^{T}\left(\tilde{\mathbf{B}} \tilde{\mathbf{B}}^{T}\right)^{-1} \mathbf{M}_{Q}^{-1 / 2}
$$

as described in [6, 5], with $\mathbf{M}_{V}$ and $\mathbf{M}_{Q}$ the diagonals of the velocity- and pressuremass-matrix, respectively, and $\tilde{\mathbf{B}}=\mathbf{M}_{Q}^{-1 / 2} \mathbf{B M}_{V}^{-1 / 2}, \tilde{\mathbf{A}}=\mathbf{M}_{V}^{-1 / 2} \mathbf{A M}_{V}^{-1 / 2}$.

5. Numerical results. In this section we present results of numerical experiments with the methods described above applied to a realistic two-phase flow problem modeled by (2.3). We consider a butanol-water and a toluol-water levitated droplet system. The geometry of the measuring cell in which the droplet is levitated in a downward going water flow is illustrated in Fig. 5.1. Its dimensions and the initial triangulation are shown in the figures 5.2 and 5.3, respectively. The $z$-axis is the symmetry axis, the $\{z=0\}$-plane is located $25 \mathrm{~mm}$ from either end. In Figure 5.4 a cross-section of a triangulation in the $\{y=0\}$-plane is shown. We use adaptive refinement near the interface.

The material properties are given in Table 5.1. The viscosity and density values in the left table correspond to a butanol (in $\Omega_{1}$ )-water (in $\Omega_{2}$ ) system under standard 


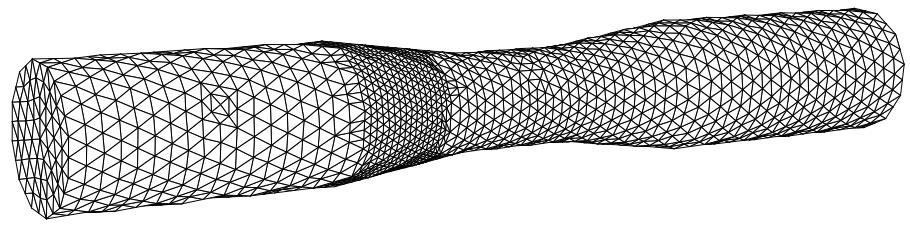

FIG. 5.3. Measuring cell with initial triangulation

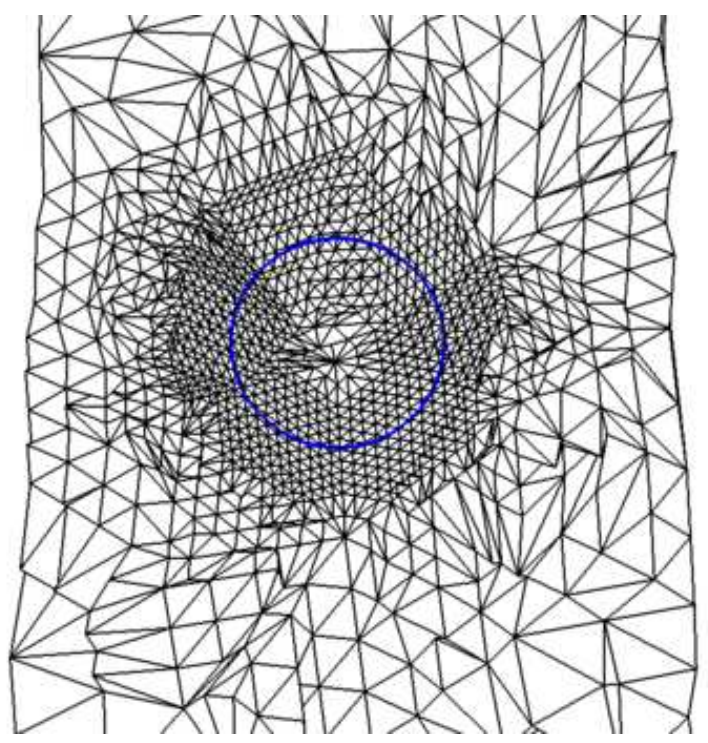

FIG. 5.4. tetrahedra, which are cut by the $y=0$-plane, $\tau=32.6 e-3 \mathrm{~N} / \mathrm{m}$; blue: droplet

conditions. On the right, the properties of the toluol-water system are shown. The smallest surface tension coefficient in the lower table is for the butanol-water system. The largest surface tension coefficient in that table corresponds to a toluol-water system. Larger surface tension coefficients induce larger localized surface tension forces which are harder to treat numerically. For all three values of the surface tension coefficient we keep the same viscosity and density values as given in the left table of Table 5.1. This in order to limit the number of different parameter values. In numerical experiments we observed that the variation of density and viscosity between the butanol-water and toluol-water system has only negligible effect on the performance of the numerical methods used.

The initial and boundary conditions are as follows. On the water inflow boundary (i.e. on the top) a quadratic velocity-profile with maximal velocity $-40 \mathrm{e}-3 \mathrm{~m} / \mathrm{s}$ is prescribed. For $\tau=1.63 \mathrm{e}-3 \mathrm{~N} / \mathrm{m}$ we use $-35 \mathrm{~mm} / \mathrm{s}$. Otherwise, the droplet drifts out of the cell. At the water outflow boundary $(z=-25 \mathrm{~mm})$ natural boundaryconditions are applied. On the remaining part of the boundary no-slip conditions for velocity are prescribed. In all experiments the initial velocity field $\mathbf{u}(\mathbf{x}, 0)$ is a priori computed by solving a stationary Navier-Stokes problem with a fixed spherical droplet of radius $1 \mathrm{~mm}$ located at $z=-6.2 \mathrm{~mm}$. For $\tau=1.63 \mathrm{e}-3 \mathrm{~N} / \mathrm{m}$ the droplet is located at $z=-10 \mathrm{~mm}$. The initial triangulation, cf. Fig. 5.3, contains 4635 tetrahedra. In the proximity of the interface local refinement is applied to obtain a local meshsize $h_{\Gamma}=0.148 \mathrm{~mm}$. The triangulation is changed and adapted to the moving interface 


\begin{tabular}{|c|c|c|c|c|c|}
\hline \multicolumn{3}{|c|}{ butanol-water } & \multicolumn{3}{|c|}{ toluol-water } \\
\hline & $\mu[P a \cdot s]$ & $\rho\left[\mathrm{kg} / \mathrm{m}^{3}\right]$ & & $\mu[P a \cdot s]$ & $\rho\left[\mathrm{kg} / \mathrm{m}^{3}\right]$ \\
\hline$\Omega_{1}$ & $3.28 \mathrm{e}-3$ & 845 & $\Omega_{1}$ & $5.96 \mathrm{e}-4$ & 867 \\
\hline \multirow[t]{3}{*}{$\Omega_{2}$} & $1.39 \mathrm{e}-3$ & 987 & $\Omega_{2}$ & $1.03 \mathrm{e}-3$ & 999 \\
\hline & & butanol & & toluol & \\
\hline & & $1.63 \mathrm{e}-3$ & $8.15 \mathrm{e}-3$ & $32.6 \mathrm{e}-3$ & \\
\hline
\end{tabular}

every ten timesteps.

In our solver we set parameters as follows. In the implicit Euler time discretization we use in each time step a weak coupling such that we only have to solve the discrete Navier-Stokes equations and the level set equation once per time step. The preconditioned GMRES iteration for the discrete level set equation is stopped if the relative error of the preconditioned residual is less than 1e-10. The relaxed defect correction method applied for linearization of the Navier-Stokes equations is stopped, if the Euclidean norm of the defect is reduced by a factor of 1e5. For the preconditioned GCR method for solving the Oseen equations we use a stopping criterion of 0.1 relative to the Euclidean norm of the defect in the Navier-Stokes solver.

Using these initializations and parameter choices we computed the dynamics of three levitated droplet systems with material properties given in Table 5.1. We used both the standard linear finite element space $Q_{h}$ and the modified XFE space $Q_{h}^{\gamma}$. In the latter we used the value $\hat{c}=0.1$, cf. (3.5). Results are shown in Fig. 5.5.

We comment on some features of the computed solutions. For $\tau=1.63 \mathrm{e}-3 \mathrm{~N} / \mathrm{m}$ we obtain a droplet in equilibrium position with both finite-element types as depicted in the top row of figure 5.5. The surface of the droplet is visibly smoother for the $\mathbf{V}_{h}-Q_{h}^{\gamma}$ pair. Figure 5.6 shows the vertical position of the barycenter of the droplet plotted over time. After similar behavior in the first $0.1 \mathrm{~s}$ the droplets evolve differently. The $Q_{h}$-droplet stabilizes at a vertical position of $-7.5 \mathrm{~mm}$, whereas the $Q_{h}^{\gamma}$-droplet ascends and stabilizes around $-6.35 \mathrm{~mm}$. Currently, there are no experimental data to verify which position is closer to reality.

For the two higher interfacial tensions we obtain equilibrium positions only with the $\mathbf{V}_{h}-Q_{h}^{\gamma}$ pair. These are shown in the right column in figure 5.5. Note the smooth appearance of the droplet surface and the symmetry of the velocity field around the droplet. The $\mathbf{V}_{h}-Q_{h}$ droplets develop spikes and are transported out of the measuring cells due to (very) large spurious velocities. For $\tau=32.6 \mathrm{e}-3 \mathrm{~N} / \mathrm{m}$ the flow field is severely disturbed. Typical numerical solutions are shown in the left column in Figure 5.5.

For the case $\tau=8.15 \mathrm{e}-3 \mathrm{~N} / \mathrm{m}$ the velocity field inside the droplet is shown in Fig. 5.7.

Stable equilibria are observed in experiments with the toluol-water system, e. g. $[1,10]$, where deuterated water was used. The $\mathbf{V}_{h}-Q_{h}^{\gamma}$ simulations match the experimental results (much) better than the $\mathbf{V}_{h}-Q_{h}$ simulations. On the left of Fig. 5.8 the velocity-distribution in the $\{y=0\}$-plane of a levitated toluol-droplet is shown as measured by a fast NMR-technique, cf. [1]. The inflow velocity is $94 \mathrm{~mm} / \mathrm{s}$. On the right, the simulation of this droplet using the $\mathbf{V}_{h}-Q_{h}^{\gamma}$-pair is shown. The inflow velocity is $88 \mathrm{~mm} / \mathrm{s}$. The droplet are ellipsoidal. In the $\{y=0\}$-plane we determined the lengths, denoted by $a$ and $b$, of the principal axes and computed the eccentricity 

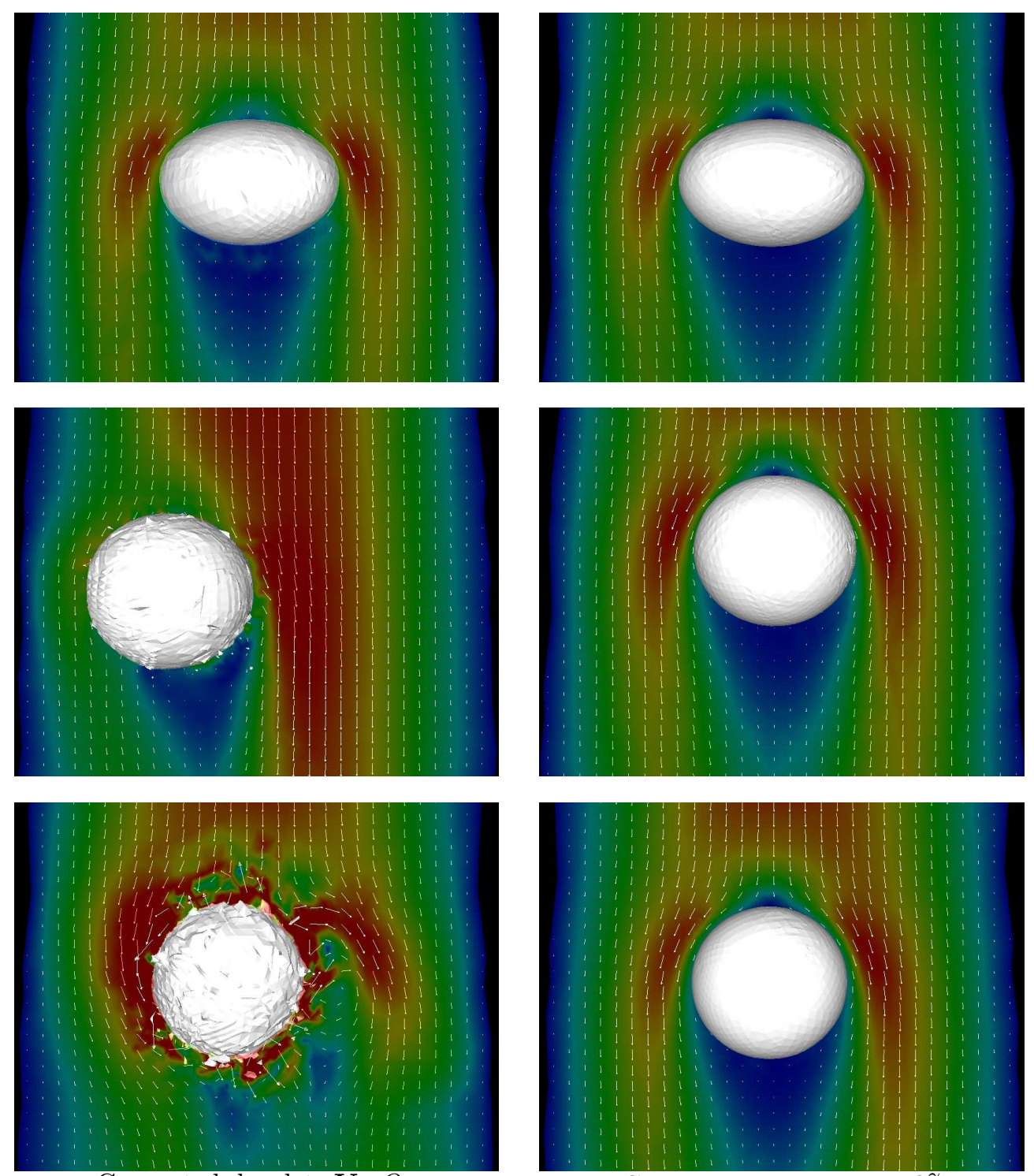

Computed droplets $\mathbf{V}_{h}-Q_{h}$

Computed droplets $\mathbf{V}_{h^{-}} Q_{h}^{\gamma}$

FIG. 5.5. From top to bottom: interfacial tension $\tau=1.63 e-3 N / m, 8.15 e-3 N / m, 32.6 e-3 N / m$

measure $\epsilon=\sqrt{1-(b / a)^{2}}$. The values are $\epsilon=0.424$ in the NMR-image and $\epsilon=0.412$ in the simulation. The position of the droplet in the measuring-cell is not known for the NMR-experiment and therefore we can not compare the locations of the stationary droplets. Note the presence of a so-called stagnant cap with very low velocities at the bottom of the NMR-image. This is caused by surface-active agents (surfactants) in the experimental setup. Surfactants lower the interfacial tension value. To account for the significant effect of this phenomenon a variable surface tension coefficient $\tau$ is used in the model (2.3). An appropriate model for this variable surface tension coefficient is not known and therefore it is chosen in simple ad-hoc manner: The stagnant cap covers the lower 10 percent of the droplet's height and the value of the surface tension 


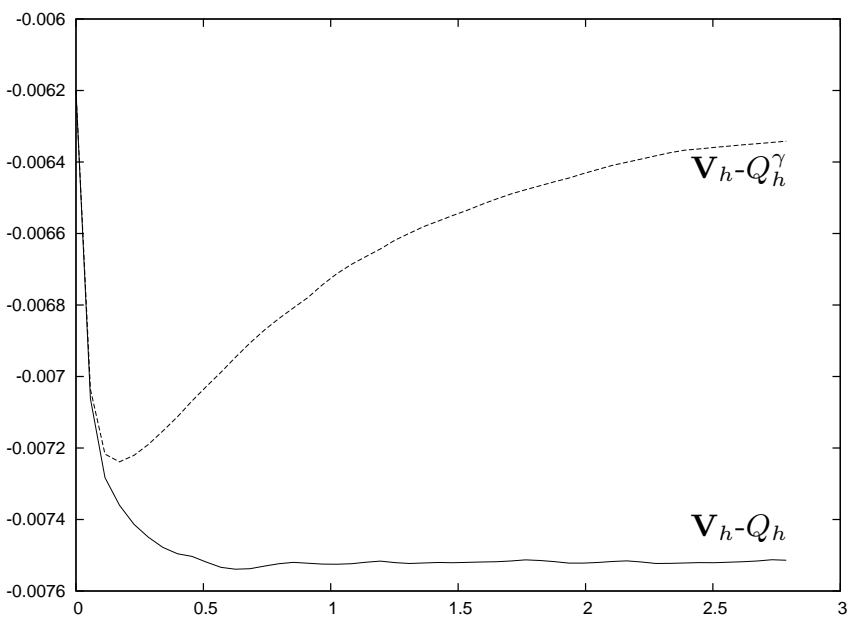

FIG. 5.6. vertical position [mm] of droplet-barycenter over time [s] for $\tau=1.63 e-3 \mathrm{~N} / \mathrm{m}$
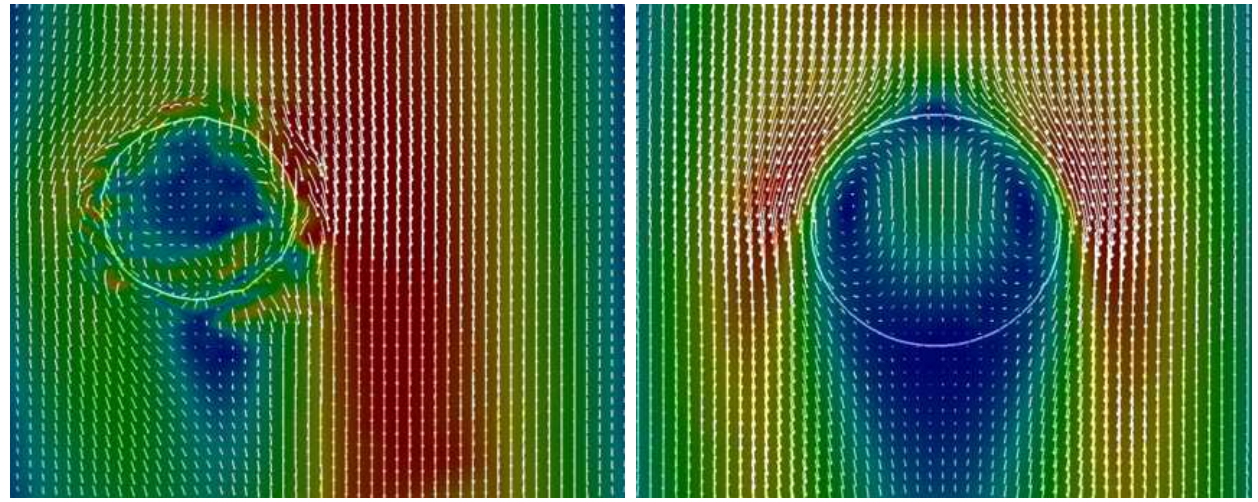

FIG. 5.7. velocity field, $y=0$-plane, $\tau=8.15 e-3 N / m$; left: $\mathbf{V}_{h}-Q_{h}$, right: $\mathbf{V}_{h}-Q_{h}^{\gamma}$

coefficient is decreased by 5 percent in this area. The numerical simulation of the model yields the result shown on right in Fig. 5.8. Note the good agreement between measurement and simulation both in the values for the eccentricity measure $\epsilon$ and in the flow field pattern. The results are obtained using the modified XFE space $Q_{h}^{\gamma}$. We were not able to produce reasonable results (on a grid with the same resolution) when the standard linear finite element space $Q_{h}$ is used for pressure discretization.

We now address the effect of the different discretization methods on the behaviour of the iterative solvers. In table 5.2 the iteration numbers of the relaxed defect correction linearization method for the Navier-Stokes equations (NS linearization) and of the preconditioned GCR method used for the Oseen equations are listed. For all interfacial tensions the iteration numbers of the linearization method are smaller for XFE than for the standard finite elements. The iteration numbers of the Oseen-solver are smaller for XFE elements with the exception of the middle interfacial tension, where they are of comparable size. We conclude, that the iterative solvers are robust with regard to the type of finite elements used for the pressure and that the better discretization method leads to lower iteration counts of the iterative solvers. For both 

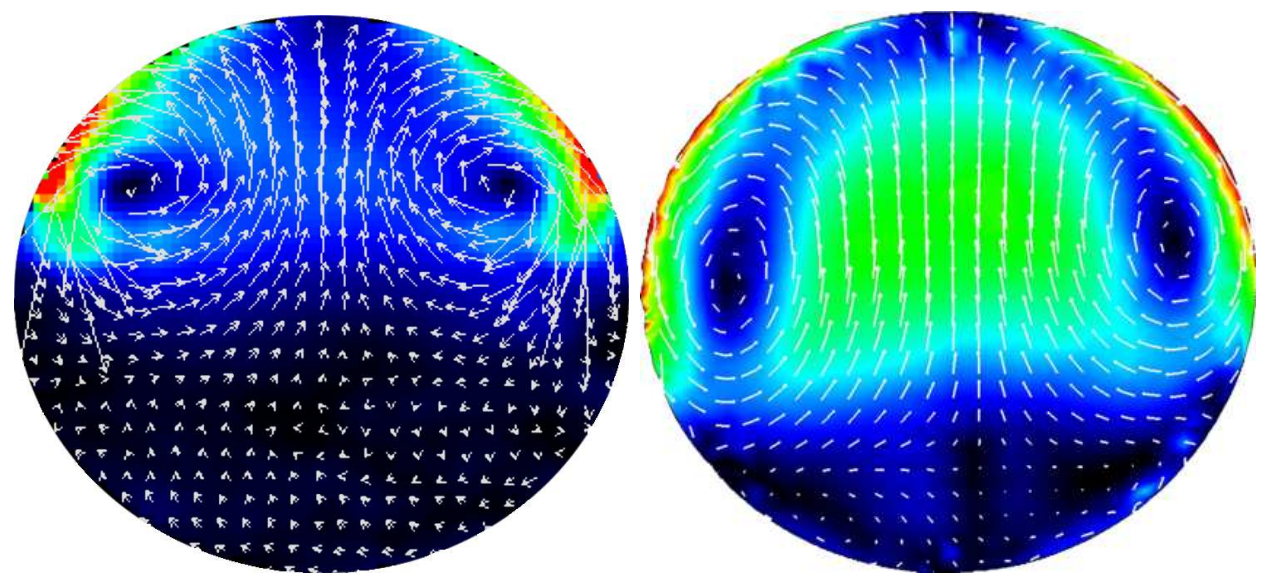

FIG. 5.8. velocity field, $y=0$-plane, toluol-droplet in water left: $N M R$-image, right: $\mathbf{V}_{h^{-}} Q_{h^{-}}^{\gamma}$ simulation

\begin{tabular}{|c|c|c|c|}
\hline pressure-FE & $\tau$ & NS linearization & Precond. GCR \\
\hline \multirow{3}{*}{$Q_{h}$} & $1.63 \mathrm{e}-3$ & 3.7 & 16.3 \\
\hline & $8.15 \mathrm{e}-3$ & 4.2 & 20.6 \\
\hline & $32.6 \mathrm{e}-3$ & 6.9 & 52.4 \\
\hline \multirow{3}{*}{$Q_{h}^{\gamma}$} & $1.63 \mathrm{e}-3$ & 3.0 & 12.0 \\
\hline & $8.15 \mathrm{e}-3$ & 3.6 & 21.6 \\
\hline & $32.6 \mathrm{e}-3$ & 4.1 & 33.2 \\
\hline
\end{tabular}

average iterations per time step (over 10 time steps at the end of the computation)

finite-element types there is a dependence on the interfacial tension: Larger values of $\tau$ make the problem more difficult for the iterative solvers.

To demonstrate the effect of the cut-off parameter $\hat{c}$ from (3.5), we compare the iteration numbers of the relaxed defect correction linearization method and of the preconditioned GCR method for different values of $\hat{c}$ for the toluol-water system in Table 5.3. For $\hat{c}=\mathcal{O}(1)$ the iteration numbers remain constant. For $\hat{c}$ below $1 \mathrm{e}-2$ the iteration numbers increase rapidly. The quality of the discrete solution, however, remains about the same for $\hat{c} \in[0,0.1]$.

Conclusions. Based on these experiments for a physically realistic two-phase levitated droplet problem we draw the following two main conclusions. Firstly, the extension of the standard linear finite element space for the pressure discretization leads to a significant improvement. This improvement increases for larger interface tension coefficients. For example, for a toluol-water system we obtain satisfactory results using the XFE pressure discretization, whereas for the standard linear finite element pressure space on the same triangulation the simulation does not yield physically realistic results. Secondly, the use of a cut-off technique in the XFE space, i.e., neglecting additional basis functions with "very small" support (as explained above), significantly improves the converge rates of the iterative solvers used. This cut-off technique is such that the good (even optimal) discretization quality is maintained. Thus we can compute accurate discrete solutions with much lower computational costs. 


\begin{tabular}{c|cc}
$\hat{c}$ & NS linearization & Precond. GCR \\
\hline $1 \mathrm{e}-0$ & 4.2 & 44.1 \\
$1 \mathrm{e}-1$ & 4.3 & 45.7 \\
$1 \mathrm{e}-2$ & 4.2 & 45.2 \\
$1 \mathrm{e}-3$ & 5.5 & 106.0 \\
$1 \mathrm{e}-4$ & 6.5 & 169.9 \\
& TABLE 5.3
\end{tabular}

toluol/water: average iterations per time step (over 10 time steps at the beginning of the computation)

Acknowledgement. The authors acknowledge the support of E. Bertakis, Chair of Thermal Process Engineering (RWTH Aachen), who provided the numerical data for Fig. 5.8 and of Dr. A. Amar, Chair of Macromolecular Chemistry (RWTH Aachen) who provided the corresponding NMR-data.

\section{REFERENCES}

[1] A. Amar, S. Stapf, And B. Blumich, Internal fluid dynamics in levitated drops by fast magnetic resonance velocimetry, Physical Review E (Statistical, Nonlinear, and Soft Matter Physics), 72 (2005), p. 030201.

[2] T. Belytschio, N. Moes, S. Usui, and C. Parimi, Arbitrary discontinuities in finite elements, Int. J. Num. Meth. Eng., 50 (2001), pp. 993-1013.

[3] J. U. Brackbill, D. B. Kothe, and C. Zemach, A continuum method for modeling surface tension, J. Comp. Phys., 100 (1992), pp. 335-354.

[4] Y. C. Chang, T. Y. Hou, B. Merriman, and S. Osher, A level set formulation of Eulerian interface capturing methods for incompressible fluid flows, J. Comp. Phys., 124 (1996), pp. 449-464.

[5] H. Elman, V. E. Howle, J. Shadid, R. Shuttleworth, and R. Tuminaro, Block preconditioners based on approximate commutators, SIAM J. Sci. Comput., 27 (2006), pp. 16511668 (electronic).

[6] H. C. Elman, Preconditioning for the steady-state navier-stokes equations with low viscosity, SIAM Journal on Scientific Computing, 20 (1999), pp. 1299-1316.

[7] S. Gross, V. Reichelt, And A. Reusken, A finite element based level set method for two-phase incompressible flows, Comp. Vis. Sci., 9 (2006), pp. 239-257.

[8] S. Gross and A. Reusken, An extended pressure finite element space for two-phase incompressible flows, J. Comp. Phys., 224 (2007), pp. 40-58.

[9] - Finite element discretization error analysis of a surface tension force in two-phase incompressible flows, SIAM J. Numer. Anal., 45 (2007), pp. 1679-1700.

[10] E. Gross-Hardt, A. Amar, S. Stapf, B. Blumich, and A. Pfennig, Flow dynamics measured and simulated inside a single levitated droplet, Industrial \& Engineering Chemistry Research, 45 (2006), pp. 416-423.

[11] R. Kimmel and J. A. Sethian, Computing geodesic paths on manifolds, Proc. Natl. Acad. Sci. USA, 95 (1998), pp. 8431-8435.

[12] N. Moes, J. Dolbow, And T. Belytschko, A finite element method for crack growth without remeshing, Int. J. Num. Meth. Eng., 46 (1999), pp. 131-150.

[13] S. Osher And R. P. Fedkiw, Level set methods: An overview and some recent results, J. Comp. Phys., 169 (2001), pp. 463-502.

[14] S. Pijl, A. Segal, C. Vuik, And P. Wesseling, A mass-conserving level-set method for modelling of multi-phase flows, Int. J. Num. Meth. Fluids, 47 (2005), pp. 339-361.

[15] S. B. Pillapakkam And P. Singh, A level-set method for computing solutions to viscoelastic two-phase flow, J. Comp. Phys., 174 (2001), pp. 552-578.

[16] A. Reusken, Analysis of an extended pressure finite element space for two-phase incompressible flows, Comp. Vis. Science, (2008). Accepted for publication.

[17] Y. SAAD, Iterative methods for sparse linear systems, Society for Industrial and Applied Mathematics, Philadelphia, PA, second ed., 2003.

[18] J. A. Sethian, Level set methods and fast marching methods, Cambridge University Press, 1999. 
[19] M. Sussman, A. S. Almgren, J. B. Bell, P. Colella, L. H. Howell, And M. L. Welcome, An adaptive level set approach for incompressible two-phase flows, J. Comp. Phys., 148 (1999), pp. 81-124.

[20] M. Sussman, P. Smereka, And S. Osher, A level set approach for computing solutions to incompressible two-phase flow, J. Comp. Phys., 114 (1994), pp. 146-159.

[21] S. TuREK, Efficient solvers for incompressible flow problems: An algorithmic approach in view of computational aspects, vol. 6 of LNCSE, Springer, Berlin, Heidelberg, 1999. 